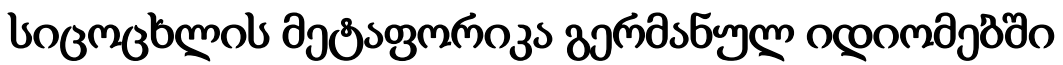

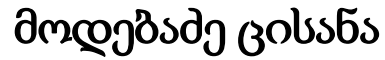

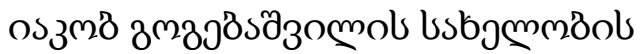

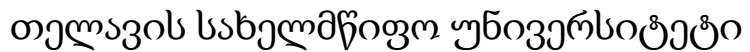

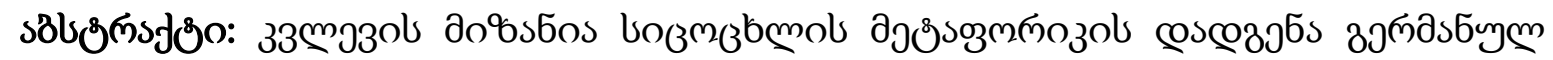

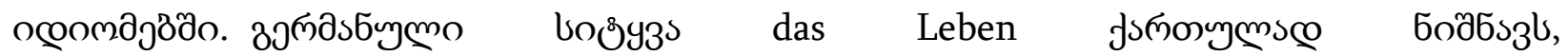

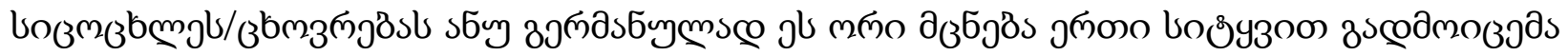

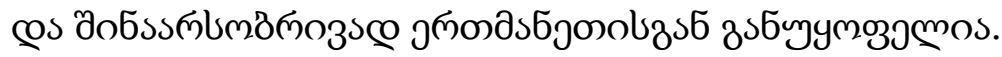

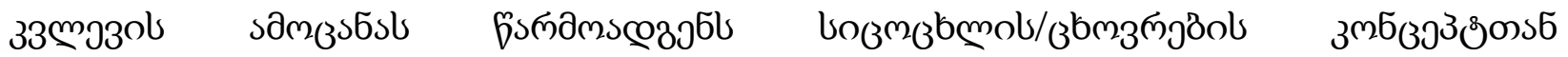

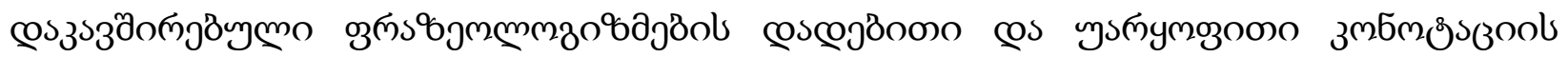

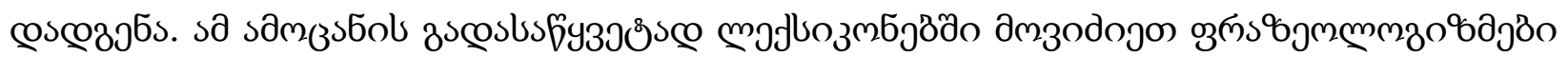

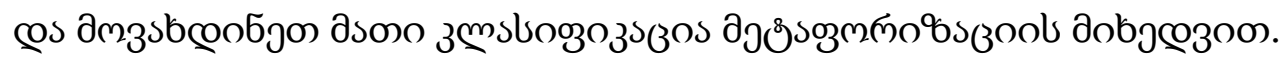

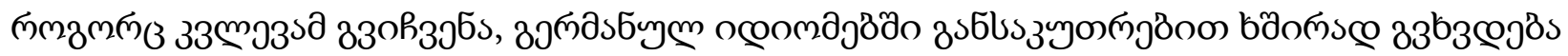

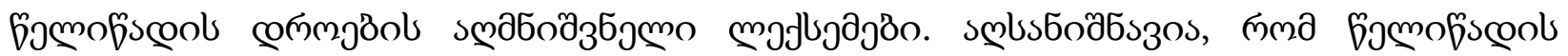

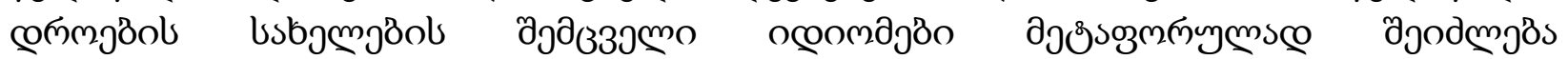

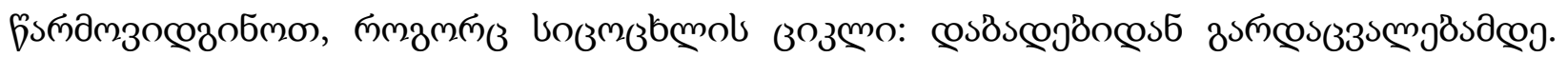

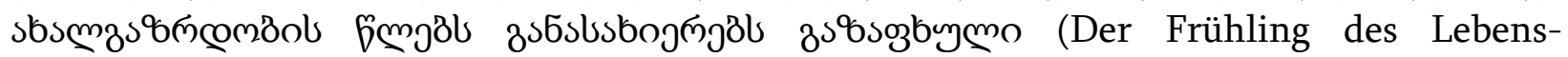

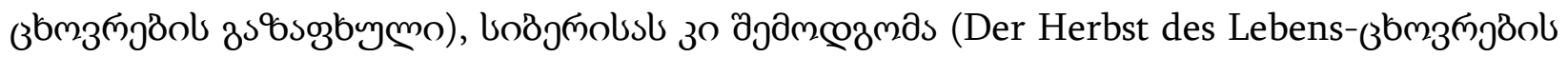

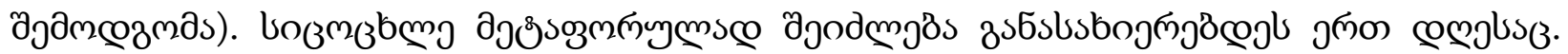

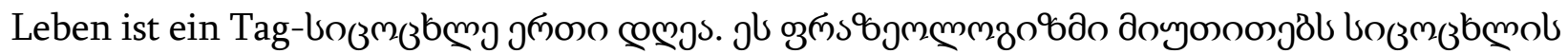

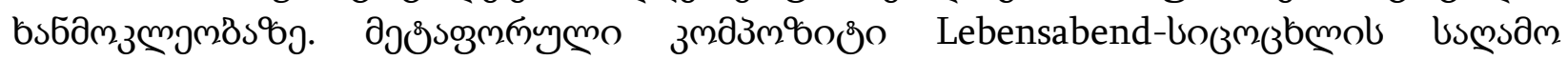

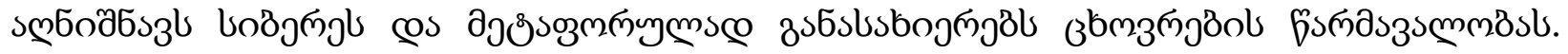

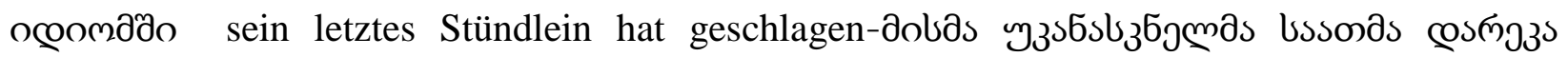

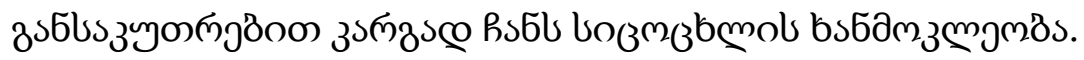

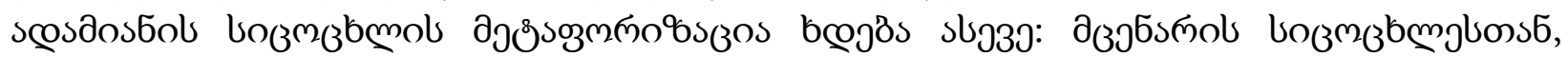

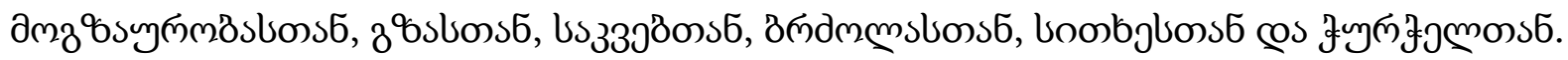

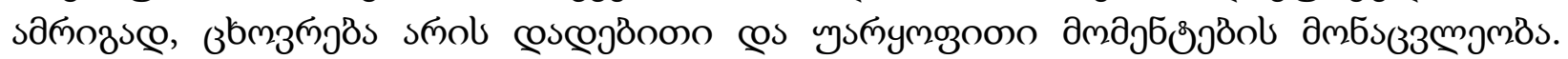

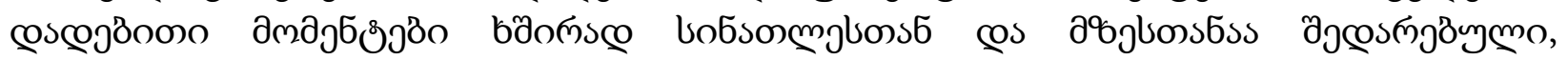

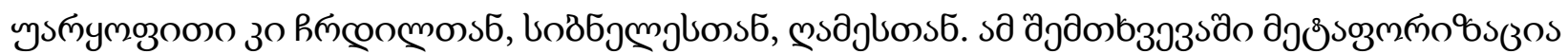

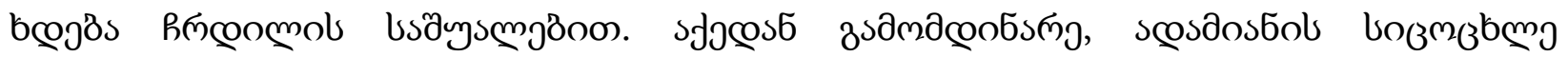

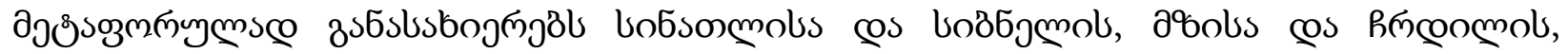

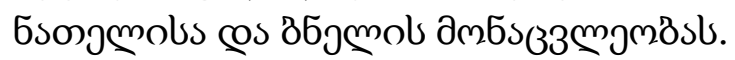

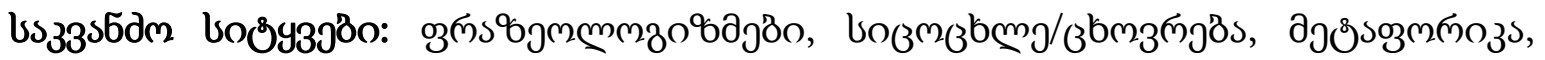

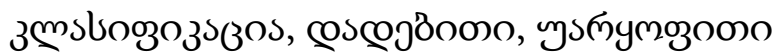

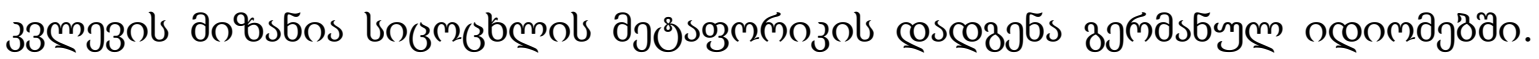




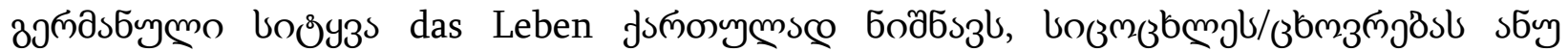

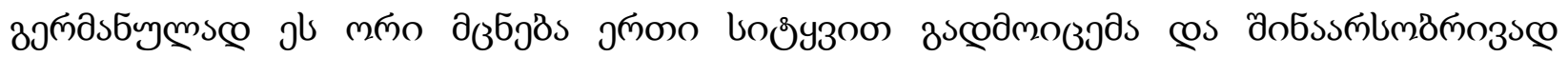

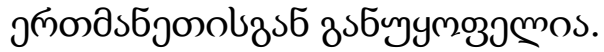

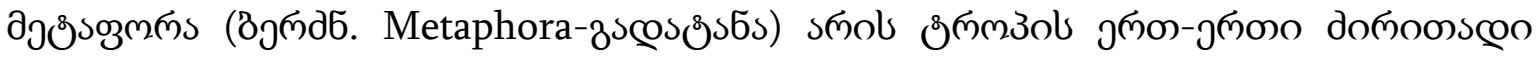

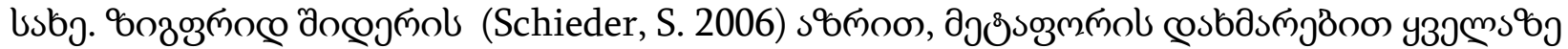

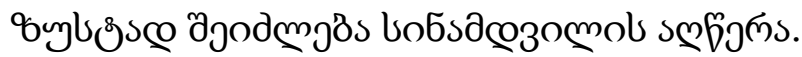

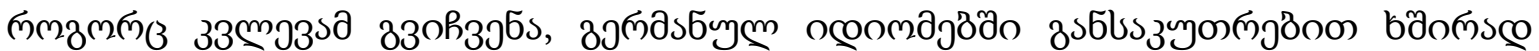

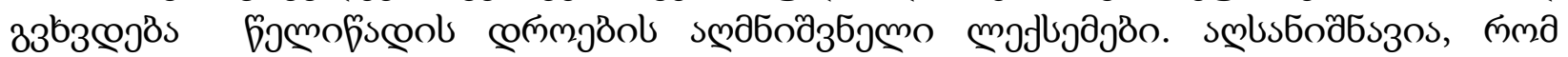

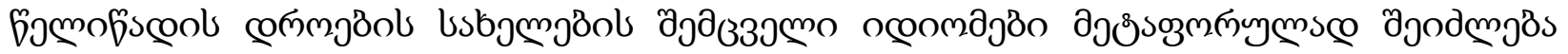

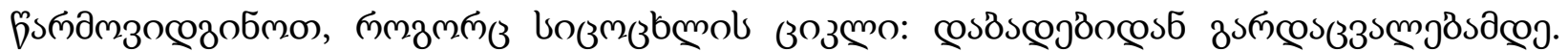

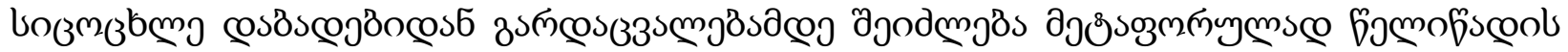

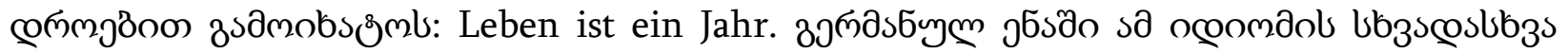

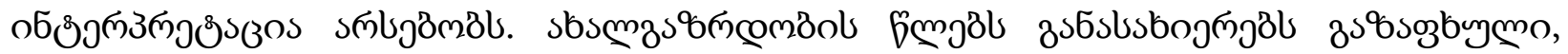

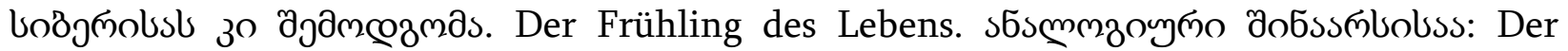

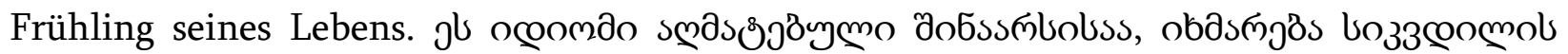

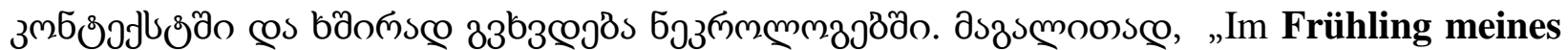
Lebens ereilte mich der Tod."

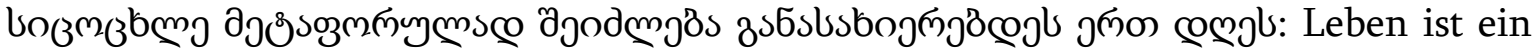

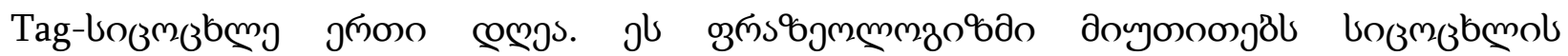

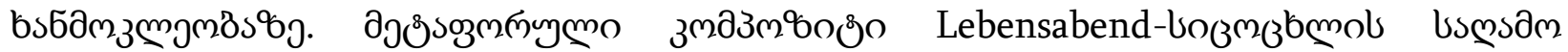

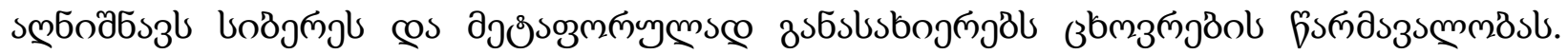

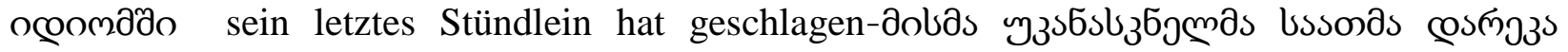

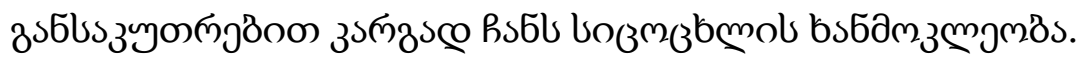

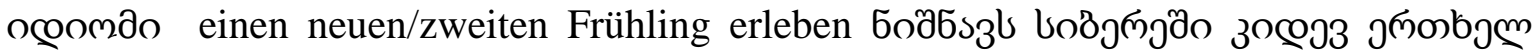

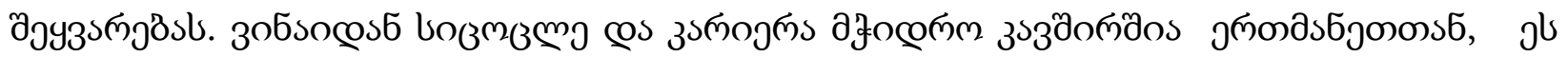

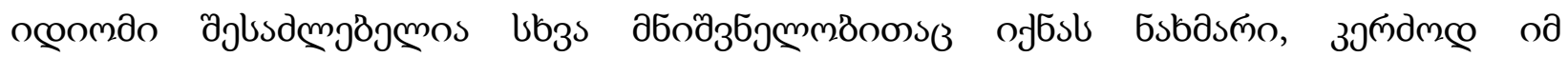

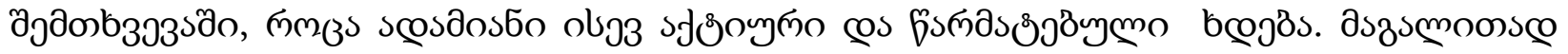
๓ме

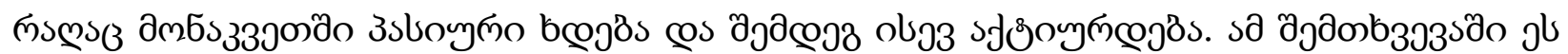

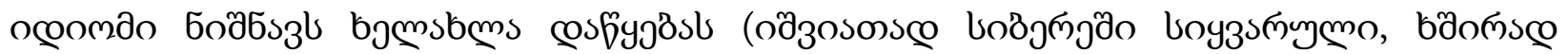

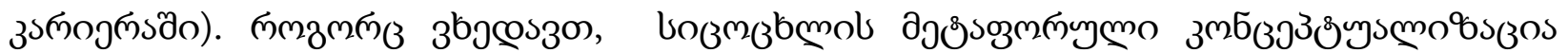

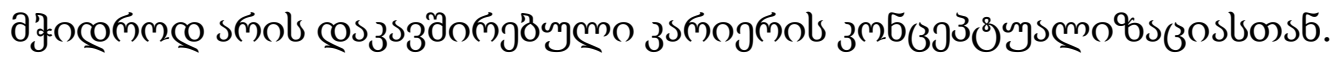

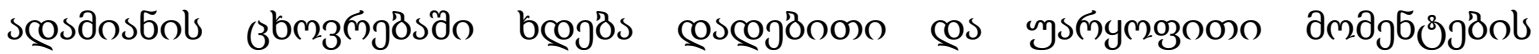

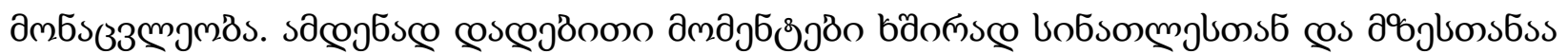

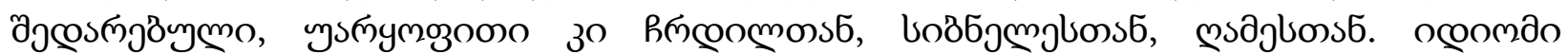

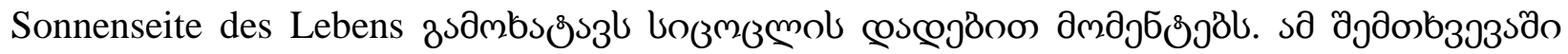

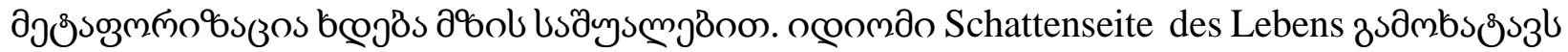

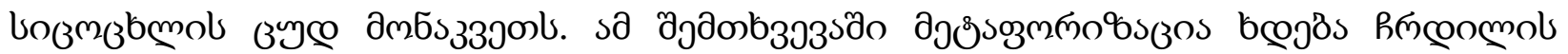

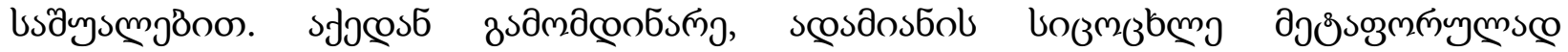

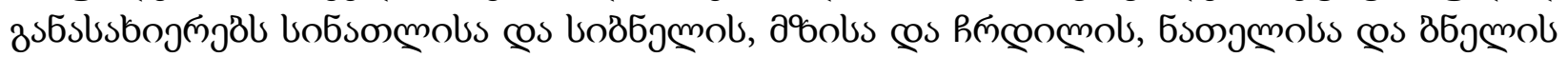
дмбs (33m)

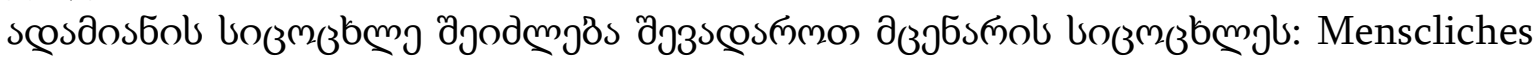




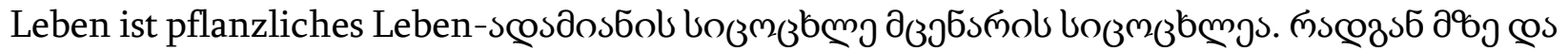

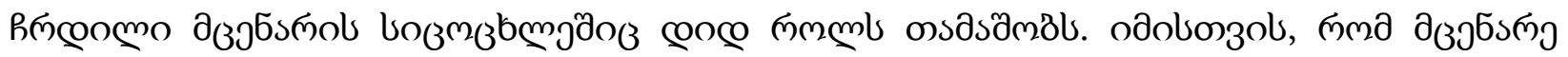

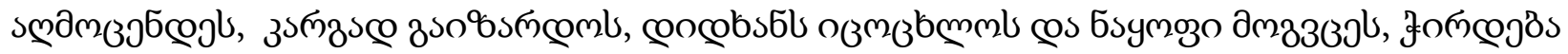

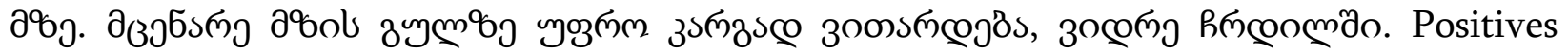

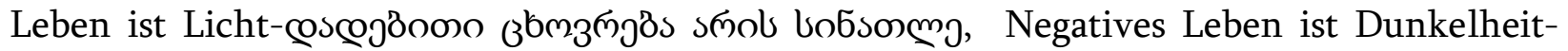

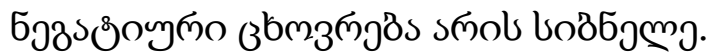

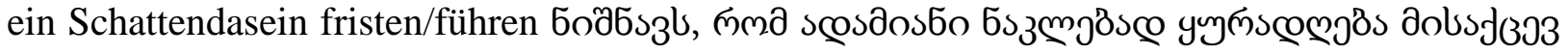
(b)

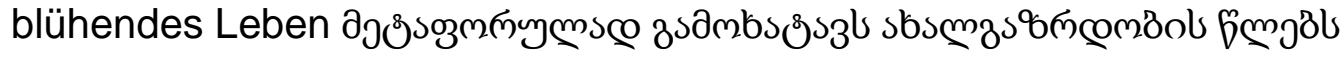

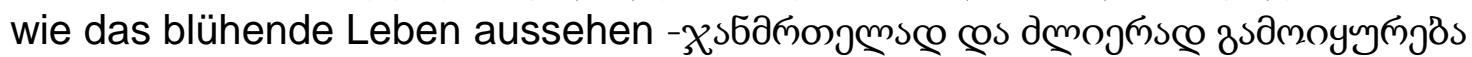

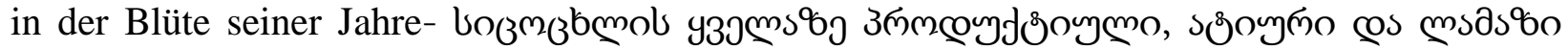

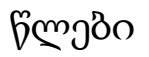

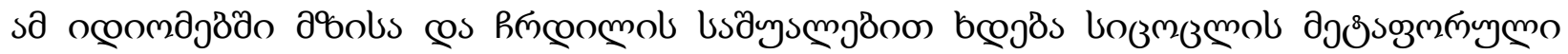

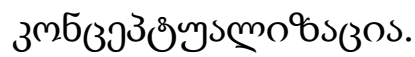

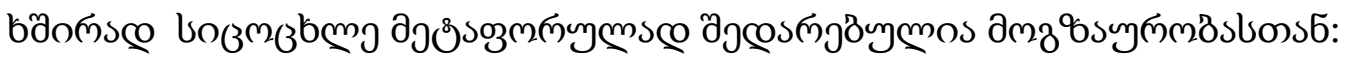

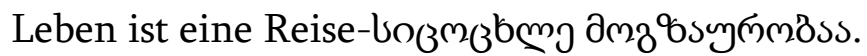

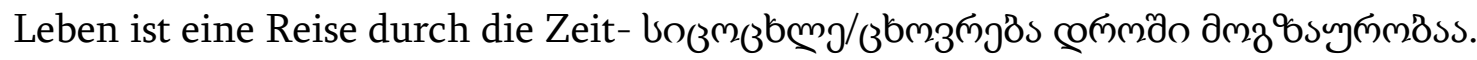

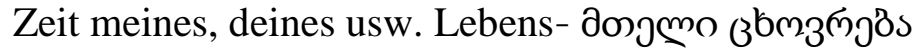

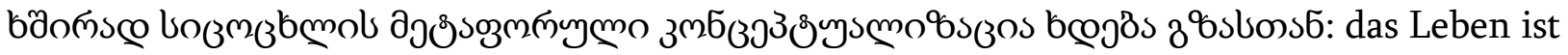

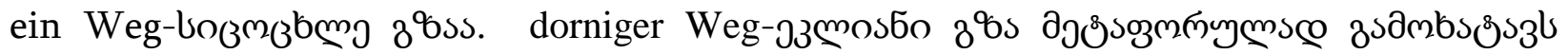

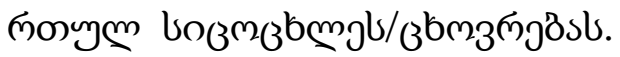

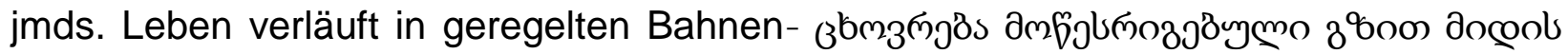

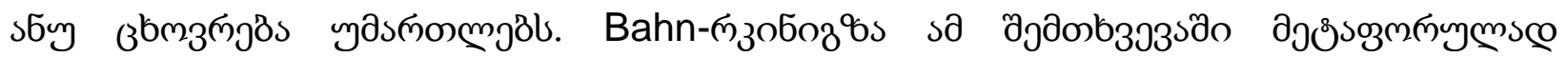

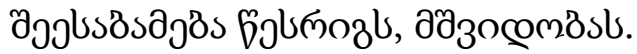

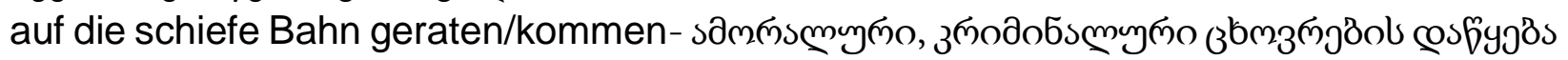

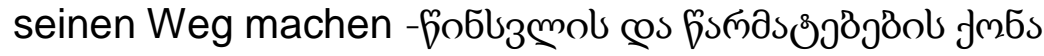

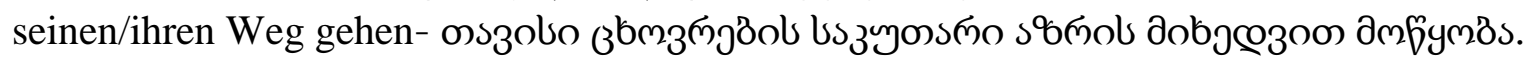

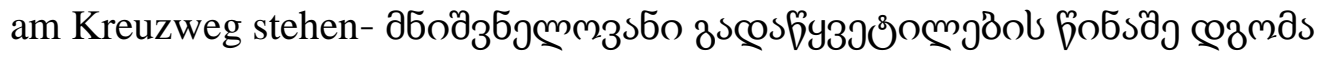

aus dem Gleis werfen/ kommen/ bringen/ springen/ geraten- Rзээmo folnozols

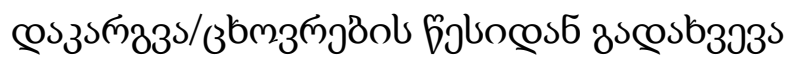

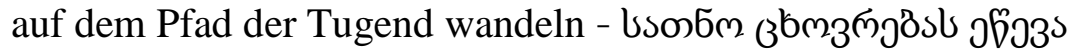

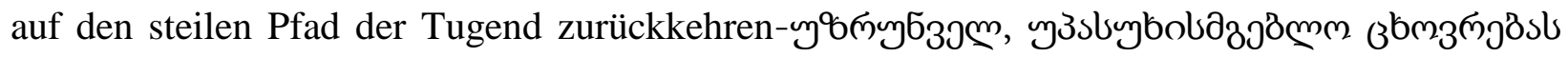
วดวง.

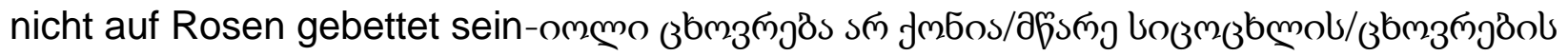
jmbs

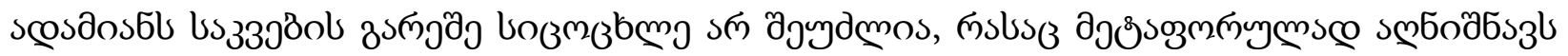

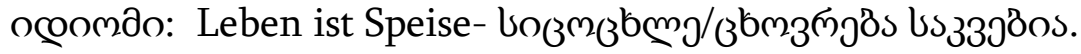

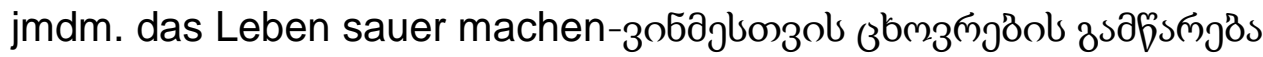

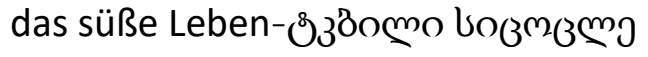

Leben ist Spiel- bozm

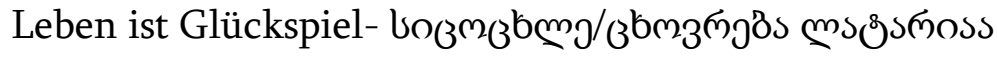

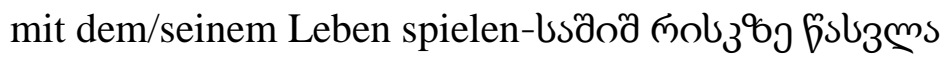


sein Leben aufs Spiel setzen- дృ

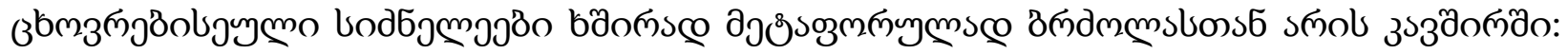
Leben ist Kampf

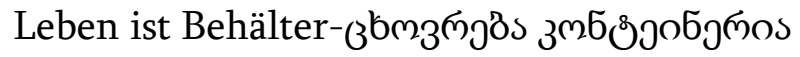

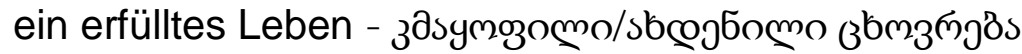

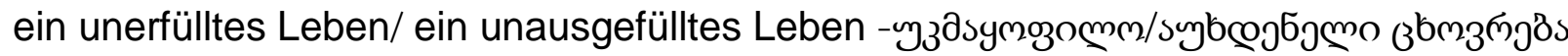
sich unausgefüllt fühlen / unausgefüllt sein- эздзумозоммю зпдбмдљ

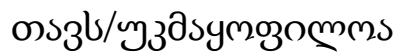

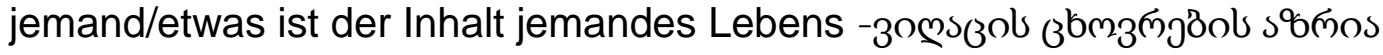

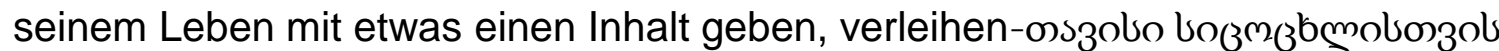

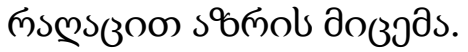

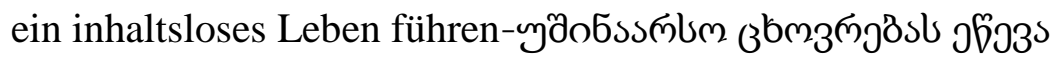

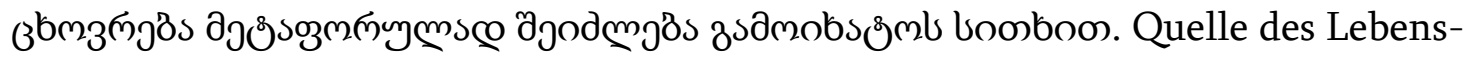

bogmabmols fysmm

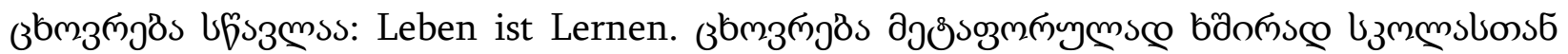

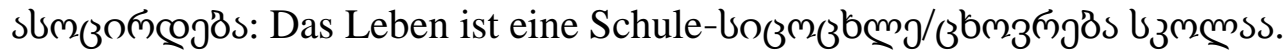

er ist in eine harte Schule gegangen, hat eine harte Schule durchgemacht-дszsmo bzmms s

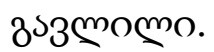

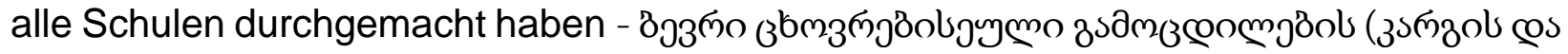
s3ob) jmbs.

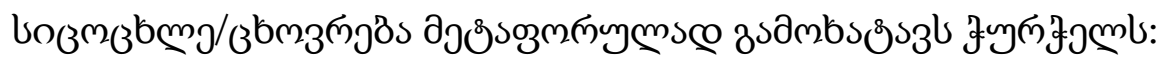

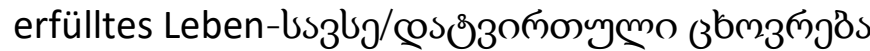

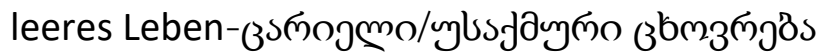

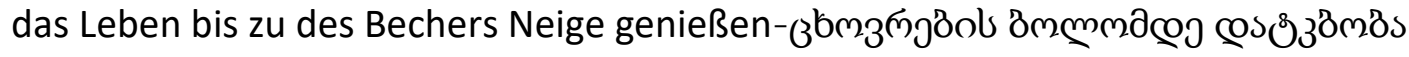

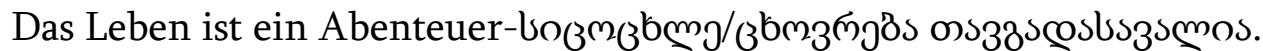

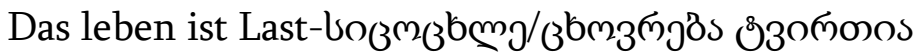

Das Leben ist ein Schauspiel-

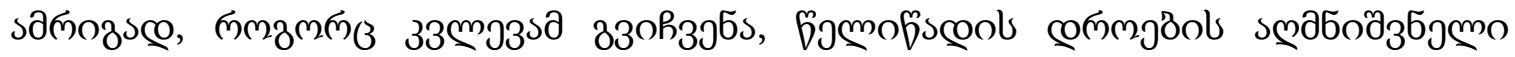

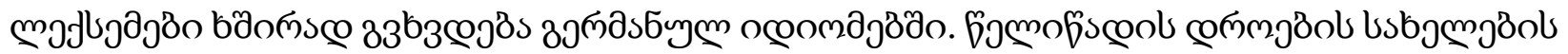

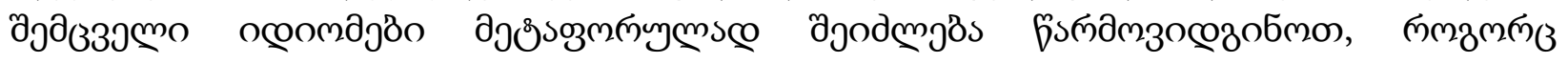

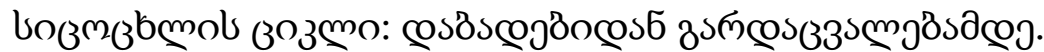

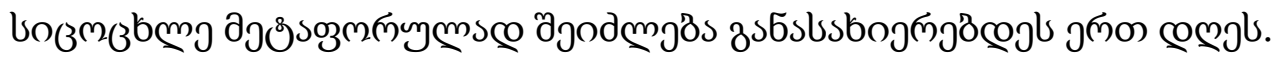

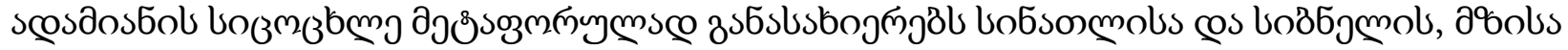

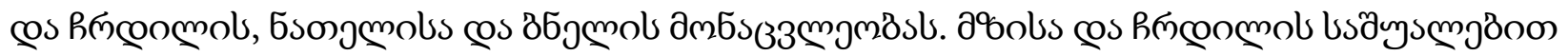

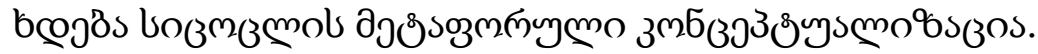

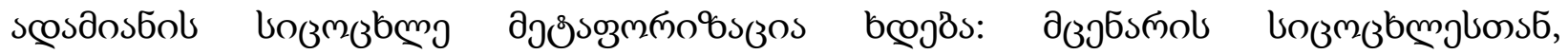

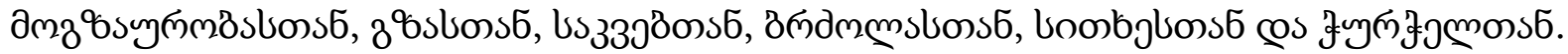

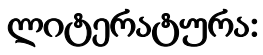

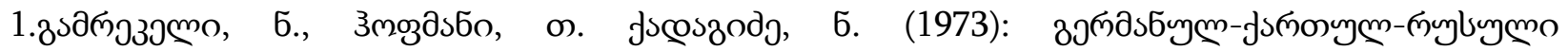

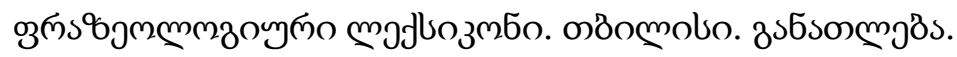




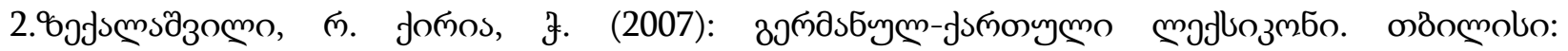

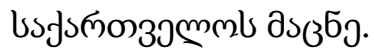

3.Götz D. u. a.:(2008) Droßwörterbuch Deutsch als Fremdsprache; Langenscheidt-Berlin- München Wien -Zürich -New York

4.Duden (Deutsches Universalwörterbuch) (5. überarbeitete Aufgabe) (2003). Dudenverlag. Mannheim. Leipzig. Wien. Zürich.

5. Duden (2008): Wörterbuch der deutschen Idiomatik. 3., Mannheim: Dudenverlag.

6. Fleischer W.(1997): Phraseologie der deutschen Gegenwartssprache.Tübingen: Max Niemeyer

7. Schieder, S. (2006): Die gestaltende Kraft von Sprachbildern und Metaphern. Trier: Universität Trier

\title{
Metaphors of Life in German Idioms
}

\author{
Modebadze Tsisana \\ Iakob Gogebashvili Telavi State University
}

\begin{abstract}
The aim of the study is to define the metaphoric meaning of the concept 'life' in the German idioms. The German word das Leben means both to live and life in Georgian. It means German does not differentiate them.

Our task is to define the positive and the negative connotations of idioms containing the concept of life/ live. To solve this task, we searched for the relevant phraseologisms in the dictionaries and classified them.

The study has revealed that lexemes denoting seasons prevail in such idioms. In a metaphoric sense, we can show these idioms as a whole life cycle: from cradle to grave. The lexeme spring stands for youth years (Der Frühling des Lebens-the spring of life), while the lexeme autumn stands for the old age (Der Herbst des Lebens- the autumn of life). In the same figurative sense, the lexeme Lebenmay refers to one day only. For instance, Leben ist ein Tag - Life is one day. This phraseologism shows the brevity of life. Metaphorical compound Lebensabend means the evening of life, that is the old age. The brevity of life is also reflected in the idiom sein letztes Stündlein hat geschlagen- 'his last hour struck', meaning, his life came to an end.

Metaphorisation of human's life is also common with the following lexemes: plants, travel, road, food, struggle, liquid and vessel.

Thus, life is alternation of positive and negative moments. The former are often compared to light and the sun, while the latter - to shade, darkness and night. Proceeding from this life is a regular alteration of light and darkness, the sun and shade, light and dark.
\end{abstract}

Key words: phraseologisms, life/live. Metaphoric, classification, positive, negative 